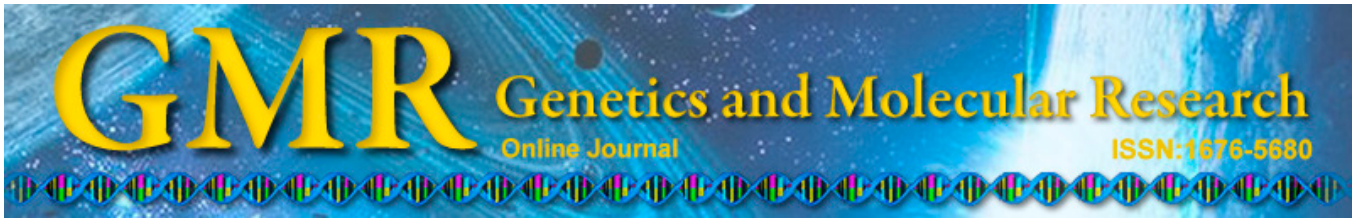

\title{
Conventional and contrast-enhanced ultrasound assessment of craniocerebral gunshot wounds
}

\author{
D. Deng ${ }^{1 *}$, G. Dan ${ }^{2 *}$, J. Tao ${ }^{1}$, X.-B. Wu ${ }^{1}$, Z. Chen ${ }^{1}$, M. Chang ${ }^{1}$, M.-S. Liao ${ }^{1}$ \\ and $\mathrm{F} . \mathrm{He}^{1}$ \\ ${ }^{1}$ Department of Ultrasound, Military General Hospital of Chengdou PLA, \\ Chengdou, China \\ ${ }^{2}$ Clinical Laboratory, Military General Hospital of Chengdou PLA, Chengdou, \\ China \\ *These authors contributed equally to this study. \\ Corresponding author: D. Deng \\ E-mail: dddoccn@163.com
}

Genet. Mol. Res. 14 (2): 3345-3354 (2015)

Received June 27, 2014

Accepted October 29, 2014

Published April 13, 2015

DOI http://dx.doi.org/10.4238/2015.April.13.14

\begin{abstract}
This study aimed to investigate the characteristic features of craniocerebral gunshot wounds by conventional ultrasound (CUS) and evaluate the efficacy of contrast-enhanced ultrasound (CEUS) in differentiation of tissue condition in wounds. Twenty crossbreed dogs (treatment: $\mathrm{N}=15$; control: $\mathrm{N}=5$ ) were used in the study. Pipe-shaped hyperechoes of varying size were found by CUS in most of the treated animals. The echoic areas were distinct from the neighboring brain tissue and did not change with time. CEUS revealed that the pipeshaped echo was unenhanced in majority of the injured brains and the surrounding tissue was either heterogeneously enhanced or unenhanced. Pathological analysis confirmed that the contrast-filling-defect area indicated necrotic tissue and the heterogeneous minimally enhanced areas indicated degenerative tissue. CUS imaging enabled detection of hematomas and CEUS indicated that the filling defect was in the center
\end{abstract}


of the hematoma, with enhancement gradually increasing towards the periphery. CUS could effectively detect a wound tract, hematoma, and the craniocerebral area injured by a gunshot, while CEUS could accurately reveal necrotic tissue in the injured area and differentiate the degenerative from normal tissue.

Key words: Conventional ultrasound; Contrast-enhanced ultrasound; Crossbreed dogs; Brain; Gunshot wound

\section{INTRODUCTION}

Craniocerebral gunshot injuries are difficult to handle; therefore, their morbidity and mortality rates are especially high. They account for the majority of battlefield and civilian gunshot casualties (Yetiser and Kahramanyo, 1998). Intracranial hematoma may occur in craniocerebral gunshot wounds caused by different weapons. These injuries not only leave a wound tract caused from tissue injury in the trajectory (i.e., the primary wound tract), but also contain necrotic or degenerative tissue and residual foreign substances in the injured area. In order to rescue craniocerebral gunshot injuries, it is important to remove foreign substances as early as possible and clear intracranial hematoma and necrotic tissue (Carey et al., 1989). One of challenges for debridement in such treatment is that clinicians can seldom accurately locate the injured area, hematoma, and necrotic tissue. Radiography and computed tomography (CT) are conventional techniques that aid diagnosis prior to surgery; a magnetic resonance imaging (MRI) stereo system or neurosurgical navigation may be applied if necessary (Lee et al., 2008).

However, cerebral edema may compromise CT observations for complicated wound tracts and the translocation of cerebral tissue may negatively impact the location of intracranial structures by neurosurgical navigation. Moreover, these techniques are expensive, complicated to operate, and difficult to maneuver. Small or medium-sized hospitals may not be equipped with such techniques, thus preventing their use (to guide debridement) in treatment of battlefield-wounds. An easy, safe, and non-invasive intraoperative technique, would be highly valuable for the treatment of intracranial injuries.

Recent developments in ultrasonography have expanded its use in the diagnosis of craniocerebral diseases, including craniocerebral injuries (Erdoğan et al., 2005). However, little has been reported on the application of ultrasound in craniocerebral gunshot injuries to date. The unique nature of craniocerebral gunshot injuries and the advantages of ultrasound suggest that such injuries may be a new field for ultrasound application, in particular at the battlefield where large equipment is not available.

We hypothesized that conventional ultrasound (CUS), as a non-invasive intraoperative technique, could detect the injured area and primary wound tract in craniocerebral gunshot injury, and locate and measure the intracranial hematoma. Recently, contrast-enhanced ultrasound (CEUS) has been used to evaluate tissue activity. For example, to assess tissue perfusion during interventional treatment of solid tumors, evaluate necrotic tissue in pancreatitis, and define the scope of the injured area in solid organs such as the liver, spleen, and kidney (Golea et al., 2010; Moschouris et al., 2010; Lv et al., 2011). Most recently, intraoperative CEUS has guided the surgical scope of intracranial occupying masses and evaluated the effect of surgery (Kanno et al., 2005; He et al., 2008). The contrast agents for CEUS - microbubbles - are pure blood pool contrast agents that do not enter the interstitial space; thus, can be used to 
visualize changes in microcirculation and perfusion of the tissue, thus objectively evaluating tissue activity and functionality (Cosgrove, 2006). It was hypothesized that real-time CEUS would distinguish necrotic tissue in the injured area and normal tissue in the intracranial hematoma. To confirm our hypotheses, we established the craniocerebral gunshot injury model in crossbreed dogs to observe the ultrasonographic characteristics of tissue in the injured area by CUS and CEUS after removing the bone flap. We then compared these with the pathological changes in the tissue in the same injured area.

\section{MATERIAL AND METHODS}

\section{Modeling gunshot injury}

Twenty crossbreed dogs weighing 29-34 kg were randomized into the treatment $(\mathrm{N}$ $=15)$ or control group $(\mathrm{N}=5)$. Animals in the treatment group were intramuscularly injected with ketamine, followed by an infusion of $3 \%$ pentobarbital sodium $(30 \mathrm{mg} / \mathrm{kg})$. Tracheal intubation was performed and connected to an air sac for assisted ventilation. Continual electrocardiogram monitoring was also performed. The left femoral vein was catheterized for infusion or CEUS. The dog was placed on the shot platform with its head fixed at the target site. When the eyelash reflex disappeared, a 53-size smoothbore weapon (Changjiang Ltd, Chonqing, China) was triggered from a distance of $2 \mathrm{~m}$ to shoot the dog in the supraorbital temporal region of the coronal plane. The steel ball bullet weighed $0.15 \mathrm{~g}$. The speed of the bullet was $400 \mathrm{~m} / \mathrm{s}$, as determined by the XL-1000 Velometer (Dongfang Ltd, Xian, China), with an absorbed energy of 12.0 joules. Shortly after the injury, a craniotomy $(4 \times 2.5 \mathrm{~cm})$ was performed in the parietal bone to provide an acoustic window to image the brain (Figure 1). The animals in the control group underwent a similar procedure, including anesthesia, tracheal intubation, ultrasound guided catheterization in the left femoral vein, and craniotomy, but without the gunshot injury. This study was carried out in strict accordance with the recommendations in the Guide for the Care and Use of Laboratory Animals of the National Institutes of Health. The animal use protocol has been reviewed and approved by the Institutional Animal Care and Use Committee (IACUC) of Military General Hospital of Chengdu PLA.

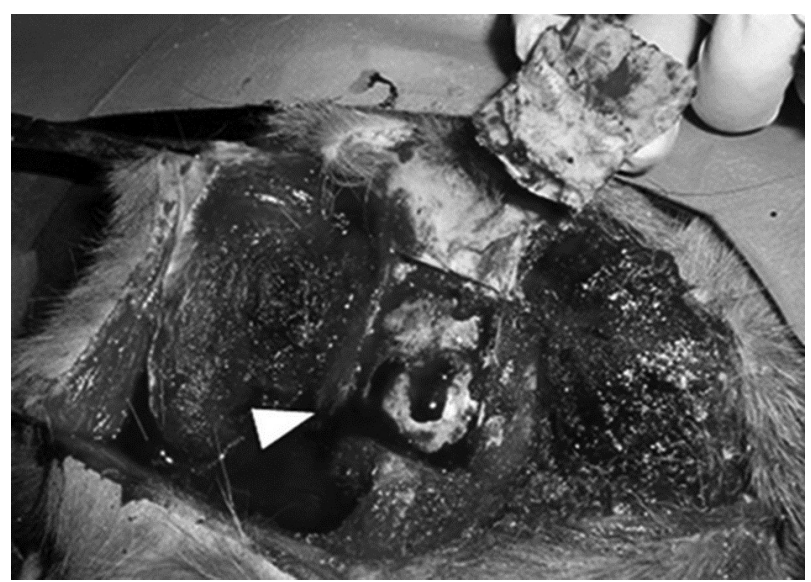

Figure 1. Frontoparietal bone window of the dog (arrowhead). 


\section{Ultrasonography}

A Philips iU22 ultrasound system (Philips Ltd., Bothell, WA, USA), with a 4-8 MHz endocavity probe $\left(\mathrm{C} 8-4_{\mathrm{v}}\right)$ and a $2-4 \mathrm{MHz}$ probe $(\mathrm{C} 5-2)$, was used to obtain $\mathrm{B}$ mode and color Doppler images. A saline bath served as the acoustic interface between the probe and dura mater through the bone window. Intracranial structures were observed and compared through 2D ultrasound and color Doppler in both groups. Findings in our previous study identified a distinguishable strong pipe-shaped echo by ultrasound for gunshot injuries in soft tissues of pigs; therefore, the intracranial injured area was traced and ultrasonographic characteristics were analyzed. Accordingly, after the injured area or hematoma was identified in the dog, the long and short axes of the entrance, middle segment, and exit of the wound tract were revealed by CEUS to observe perfusion in the wound tract and surrounding tissues. When an intracranial hematoma was found, perfusion was observed at the maximum section of the hematoma. In the control group, CEUS was also performed to observe cerebral perfusion. A C $8-4_{v}$ endocavity probe (frequency $4-8 \mathrm{MHz}$ ) was used for CEUS. A pulse-inversion contrast-imaging mode with a low mechanical index (MI 0.06-0.09) was adopted for CEUS examination. The software installed on the system enable dual display of contrast enhanced and unenhanced images. The contrast agent, SonoVue ${ }^{\circledR}$ (Bracco Imaging, Milan, Italy) is a second-generation contrast agent and has been marketed in China since 2004. It is made of stabilized microbubbles containing sulfur hexafluoride gas covered by a phospholipid membrane, with an average bubble diameter of $2.5 \mu \mathrm{m}$. SonoVue ${ }^{\mathbb{B}}$ lyophilized powder was reconstituted with $5 \mathrm{~mL}$ normal saline and vortexed for later use. Five milliliters of the suspension was rapidly bolus-injected in the femoral vein, followed by $5 \mathrm{~mL}$ of normal saline. CUS and CEUS were performed at 0,1 , and $3 \mathrm{~h}$ after craniotomy.

\section{Examination of pathology}

If distinct enhancement areas (such as unenhanced, heterogeneously distributed minimally enhanced, highly enhanced, and relatively homogeneously enhanced) were noted in the treatment group, CEUS-guided biopsies were performed in these areas using an $18 \mathrm{G}$ biopsy needle. The sectioned tissues were fixed in $10 \%$ in neutral formalin, embedded in paraffin, stained with hematoxylin and eosin (H\&E), and observed under a light microscope.

The animals were sacrificed to collect the whole brain. When the injured area was identified under ultrasound, the wound tract was incised along the long axis to observe macroscopically. Cross sections of the entrance, middle segment, and exit of the wound tract along the short axis (including the wound tract and surrounding tissue) were harvested, fixed in 10\% neutral formalin, embedded in paraffin, stained with H\&E, and observed under a light microscope. Matching samples were collected, treated, and observed in the control group.

\section{RESULTS}

\section{General information}

Two dogs died of respiratory failure because of tracheal intubation 15 min after injury. The other thirteen dogs had 100\% survival by $6 \mathrm{~h}$. 


\section{Ultrasound findings}

CUS showed a pipe-shaped hyperecho between the entrance and exit of the wound tract in most treatment animals (Figure 2a) and an L-shaped hyperecho in some treatment animals (Figure $2 \mathrm{~b}$ ). The echo was distinct from the surrounding cerebral tissue and did not change over time. The diameter of the tracts varied from $0.5-1.5 \mathrm{~cm}$ and was largest at the middle segment or exit. A heterogeneous hypoecho was noted in surrounding tissues along the pipe-shaped echo. Color Doppler revealed low blood flow signals at the periphery of the wound.

Intracranial hematoma was noted in 5 of $13 \mathrm{dogs}(35 \%)$. A heterogeneous oval masslike hyperecho was detected in the cerebral tissue with a diameter of 1.3-1.5 cm (Figure 2c). This was distinguished from the surrounding cerebral tissues. An irregular weak echo was observed inside some of the strong mass-like echoes. Color Doppler detected blood vessels around the hematoma but no definitive flow signals inside the lesion. The brain midline and surrounding cerebral tissues were translocated to different extents due to compression induced by different lesions. CUS showed no apparent changes in the wound tract and the hematoma at 0,1 , and $3 \mathrm{~h}$ after injury.

Strong echoes of steel ball bullets were noted at the exit of the wound tract or the parenchyma of the middle segment, accompanied by an echo tail in some animals (Figure 2d). Irregular hyperecho of bone flaps followed by an echo shadow was clearly defined near the wound entrance or inside the wound tract (Figure 2e). CUS showed no abnormal echo in the control group.

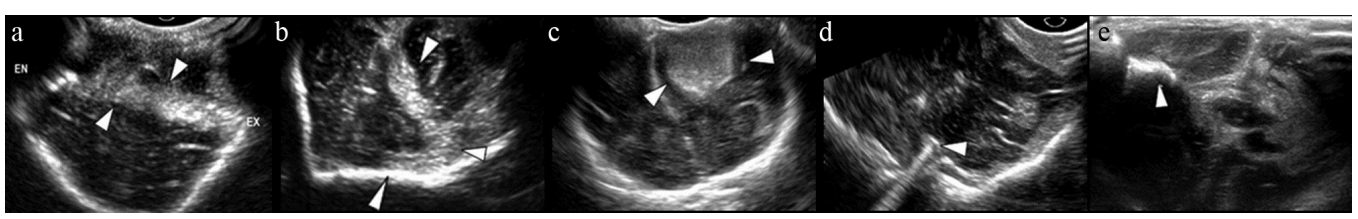

Figure 2. Two-dimensional (2D) ultrasound images of the craniocerebral gunshot wound of a dog. a. A pipeshaped hyperecho was seenbetween the entrance and exit of the wound tract (arrowhead; En: entrance; Ex: exit). b. A cone-shaped hyperecho (arrowhead) was detected. c. A roughly heterogeneous oval hyperechoic mass was revealed (arrowhead). d. Hyperecho of a steel ball bullet was noted in the parenchyma, accompanied by an echo tail (arrowhead). e. Hyperecho was noted for bone debris in the parenchyma, accompanied by a posterior echo shadow (arrowhead).

\section{CEUS}

CEUS was used to further assess the perfusion of the wound tract and the surrounding tissues. It was noted that the pipe-shaped echo patterns observed with CUS were mostly unenhanced, manifesting as a contrast filling defect (Figure 3a). Some of the lesions were partially unenhanced, characterized by heterogeneous or low contrast filling (Figure 3b). Minimally enhanced or unenhanced areas coexisted around the strong pipe-shaped echo (Figure 3c). Highly enhanced bulky blood vessels crossing the filling defect area were observed in some animals. A highly enhanced area was seen around these filling defects and heterogeneous minimally enhanced areas. A circular hyperecho was revealed in the cross-section of the short axis of the pipe-shaped echo (Figure 3d).

The contour of the hematoma was clearly depicted in CEUS. CEUS showed that the 
filling defect was in the middle of the hematoma or to one side, with enhancement gradually extending towards the periphery and having no clear boundaries (Figure 3e). Hyperenhanced blood vessels were noted to cross or penetrate the filling defect areas (Figure 3f) in some treatment animals. Some unenhanced areas were circled by highly enhanced blood vessels in the early phase of enhancement. CEUS revealed no abnormal enhancement or filling defects in the control group.

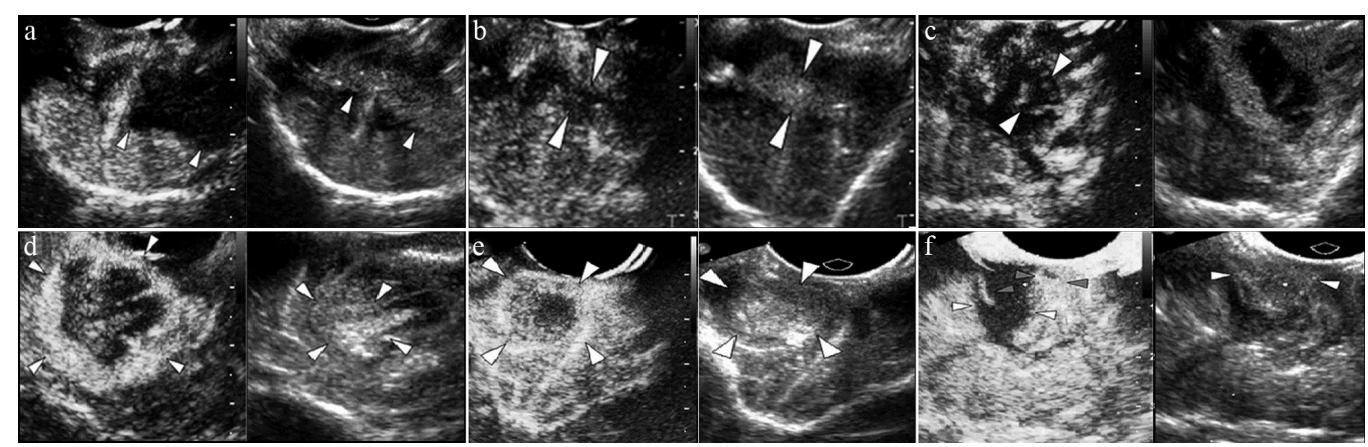

Figure 3. Contrast-enhanced ultrasound (CEUS) images show the perfusion of the craniocerebral wound tract and the surrounding tissue. a. The pipe-shaped echo was nearly unenhanced, extending towards the surrounding tissue (arrowhead). b. The pipe-shaped echo was unenhanced or heterogeneous and minimally enhanced (arrowhead). c. The adjacent area of the pipe-shaped echo was unenhanced or minimally enhanced (arrowhead). d. A corresponding circular hyperenhanced zone was demonstrated at the outside (arrowhead). e. There was a core filling defect with enhancement gradually extending to the periphery in a gradient manner (arrowhead). f. The mass contained an unenhanced area (white arrowhead) and a relatively enhanced area (yellow arrowhead), with hyperenhanced blood vessels crossing in the unenhanced area (green arrowhead).

\section{Pathological findings}

Open craniocerebral injuries from the left to the right were noted in 13 treatment animals. The exit and the middle segment of the wound tract were larger than the entrance and other parts of the wound tract (length: $0.5-1.0 \mathrm{~cm}$; Figure 4a). Penetrating and blind gunshot injuries were noted in five and eight treated animals, respectively.

The filling defect revealed by CEUS indicated necrotic or severe degenerative tissue without regular structures. Cell rupture, cytoclasis, or parenchyma swelling, disintegration, liquidation, or fusion was noted with irregular red staining under a light microscope (Figure $4 b)$. The lowly and heterogeneously enhanced areas mainly contained degenerative tissue that appeared as diffusive cell swelling, deeply stained nuclei, capillary congestion and microembolism, and limited inflammatory cell invasion with patchy necrotic tissues (Figure 4c). The highly enhanced areas were characterized by dilatation and congestion of small blood vessels in the cerebral parenchyma (Figure 4d).

Tissue was collected through incision from the wound tract and hematoma in the treatment animals. Under the light microscope, irregular necrotic tissue area, degenerative tissue area, and normal tissue area were observed without clear boundaries around the wound tract. Blood clots were found in the center of the hematoma with red irregular material containing ruptured, disintegrated, and swollen cells or tissue (Figure 4e). Blood clots were also surrounded by condensed nuclei and swollen cytoplasm that was plainly stained, as well as small 
vascular dilation, congestion, and inflammatory cell infiltration. Pathological examination revealed no abnormity in the control group (Figure 4f).

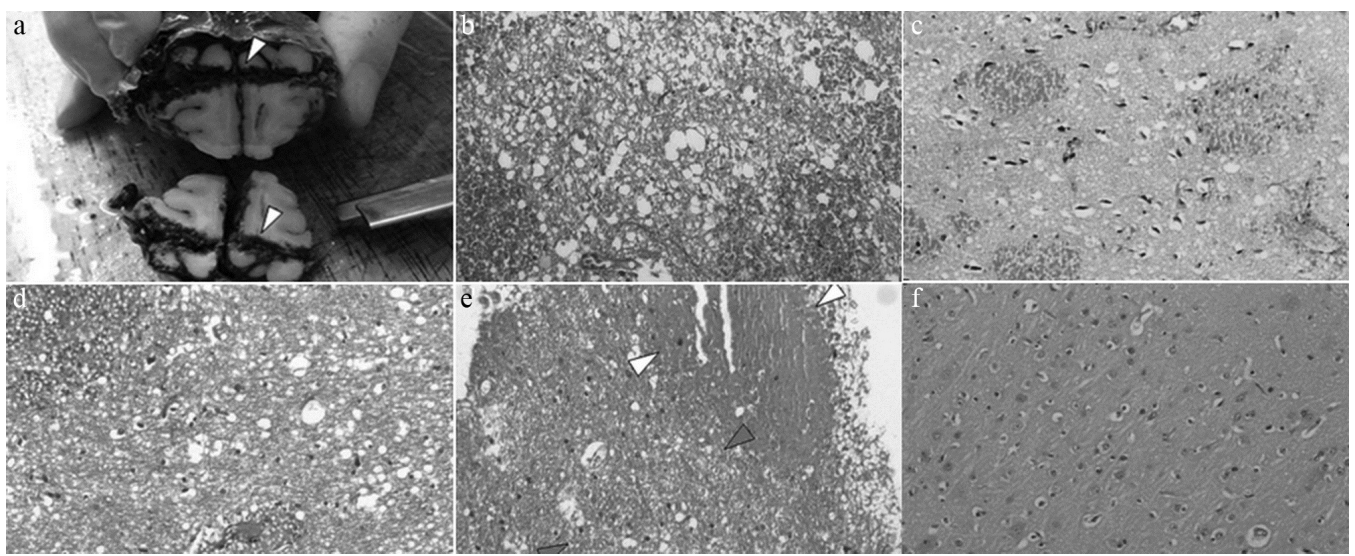

Figure 4. Craniocerebral wound tract and micrograph analysis with H\&E stain, compared with normal parenchyma tissue from a control dog. a. Macroscopic findings of the wound tract. b.-e. A representative micrograph of the different enhanced areas (original magnification, 200X). b. The unenhanced area of the pipe-shaped echo containing irregular or severe necrotic tissue. c. The relatively enhanced area of the pipe-shaped echo containing swollen cells, capillary congestion and microembolism, and limited inflammatory cell invasion with patchy necrotic tissue. d. The outer circular hyperenhanced area of the pipe-shaped echo characterized by small vascular dilation and congestion. e. The maximum area of the hematoma, showing blood clots (white arrowhead) and a degenerative area (yellow arrowhead). f. The normal parenchyma of a control dog.

\section{DISCUSSION}

The mechanism of injury from a craniocerebral gunshot is complicated. The highspeed bullet induces direct injures on intracranial structures, while the heat effect, temporary cavitation effect, and pressure from the bullet also directly or indirectly threaten surrounding cerebral tissues along the wound tract (Carey et al., 1989). Thus, it has unique pathologic characteristics in contrast with an incision or stab wound.

The craniocerebral gunshot wound generally consists of three areas, which from the center to the periphery, are the primary wound tract, contusion area, and concussion area (Carey et al., 1989). This was confirmed in our study. The primary wound tract and contusion area often contain cerebral tissue debris, blood clots, metal remnants, and hair. It is recommended that these necrotic tissues be cleared completely. The concussion area mainly contains degenerative tissue that may survive and thus, should be retained. The intracranial hematoma should be cleared because of its space-occupying effect. Bone debris and metal remnants should be removed to reduce underlying complications and adverse events. Currently, the identification of necrotic tissue during intracranial surgery relies on the experience and observation of the clinician, color and density of the tissue, and whether there is capillary bleeding. This seemingly simple approach may cause undesired injuries and complications. Thus, a simple, effective, and intraoperative technique to locate the craniocerebral gunshot wound, intracranial hematoma, foreign substances, and identify necrotic tissue is particularly valuable in clinical practice, especially when important neurological function is vulnerable. 
Currently, there are multiple imaging techniques for craniocerebral gunshot wounds, but all of them are subject to limitations. Cephalometric radiographs cannot provide adequate information about the wound tract, hematoma, and soft tissues (Gervaise et al., 2010). Cerebral angiography is an invasive diagnostic method; therefore, case selection must be careful (Dawkins et al., 2007). MRI is not recommended for use in the acute phase of craniocerebral gunshot injuries. In addition, metal debris in the brain may be removed under a strong magnetic field, leading to injury to intracranial structures, and other techniques are not compatible with MRI (Kim et al., 2002; Kühne et al., 2007). High cost and low mobility also limit the use of CT and MRI during surgery. Transcranial Doppler is mainly used to evaluate large blood vessels but not microcirculation and perfusion of tissue (Oertel et al., 2002; Ract et al., 2007). The 3D stereo system and neurosurgical navigation are not real-time techniques; therefore, cannot accurately locate intracranial lesions when translocation of tissue occurs (Benvenuti et al., 2005; Jannin and Morandi, 2007).

This study indicates that CUS, in combination with CEUS, may be of clinical value for craniocerebral gunshot injuries when the bone flap is removed. Firstly, the pipe-shaped hyperecho in cerebral tissues from CUS is a typical ultrasonographic feature of gunshot wounds that can be easily localized and identified. Because the bullet may bounce back when hitting the contralateral cranium, the wound tract may have tortuous or more complicated patterns, adding to difficulties in surgery. This study shows that CUS may be a simple and effective technique not only for locating the injured area, wound tract, and extent of injury, but also effectively detect and identify intracranial hematoma and foreign substances to guide surgical clearing.

This study also demonstrates that CEUS can accurately evaluate the activity and functionality of the surrounding tissues and hematoma with contrast perfusion. In this study, the contrast filling defect seen under CEUS examination indicated necrotic tissue, including in the primary wound tract and contusion area, while heterogeneous minimally or slowly enhanced areas (mainly in the concussion area) indicated majority of the degenerative tissue. The circular, highly enhanced area in the periphery of the wound tract was the boundary between the injured area and surrounding tissue. It is noteworthy that the scope of the strong pipe-shaped echo under CUS was not consistent with the filling defect under CEUS. For example, the area of the strong pipe-shaped echo contained mostly unenhanced necrotic tissue but also some partially viable tissues. The contrast filling defect on CEUS also revealed the presence of necrotic tissue outside this area. This suggested that accurate debridement could not be performed using only examination with 2D unenhanced ultrasound (CUS) and CEUS would be very useful in this condition. As a real time technique, it guides neurological surgeons during careful debridement of necrotic tissue and evaluation of surgical effects, thus reducing postoperative time and improving prognosis. The presence of hyperenhanced blood vessels crossing the filling defect area found on CEUS suggests a potential risk of massive bleeding if no caution is exercised in the debridement procedure.

CEUS showed that the filling defect mainly occurred in the core of the hematoma with enhancement gradually extending to the periphery. Hyperenhanced blood vessels also crossed the filling defect, as revealed by CEUS. However, CUS did not find definite abnormal echoes near the hematoma although filling defects were observed in CEUS. Thus, a radical cleaning of the hematoma revealed by CUS might not be necessary because some partially viable tissue may be retained, while some "normal" tissue revealed by CUS may be necrotic and require total debridement.

CEUS has the following potential advantages for diagnosis and management of craniocerebral gunshot wounds. It is simple, effective, rapid, and non/minimally invasive. In par- 
ticular, necrotic tissues of craniocerebral gunshot wounds may be evaluated intraoperatively at the bedside or battlefield with small portable handy ultrasound machines. Although the ultrasound contrast agent needs to be injected intravenously, CEUS can be easily performed by a trained sonographer in a short time. CEUS could be a better technique to track tissue perfusion when compared to enhanced CT or MRI, because microbubbles are a pure blood pool agent of micrometers in size; therefore, they do not extravagate from the blood vessel. CEUS could help neurological surgeons to prevent damaging major tissues and blood vessels, reducing surgical time and risk. CEUS may also facilitate the targeted insertion of a biopsy needle and reduce cerebral injuries, in addition to procedural duration.

$\mathrm{CT}, \mathrm{MRI}$, and positron emission tomography can primarily be used to distinguish irreversible injuries and viable tissue (Zimmer and Reith, 2008; Metting et al., 2009). MRI diffusion- and perfusion-weighted imaging are the most rapid and accurate techniques (Mitsuya et al., 2010). However, for patients who are immobile and those with acute craniocerebral gunshot injuries, CEUS prove valuable in the absence of MRI or when rapid intervention is necessary.

There are some limitations in this study. Although the results are representative, the number of animals is relatively small. Moreover, the bone flap needs to be removed as CEUS requires using low mechanical index (MI) ultrasound energy in order to avoid microbubble destruction. Moreover, this study is only preliminary and qualitative, thus future pre-clinical and clinical evaluation is required to confirm our findings.

As a noninvasive bedside and intraoperative technique, CUS can be used easily and effectively to detect the injured area and the wound tract of craniocerebral gunshot injuries. The wound tract is generally revealed as a pipe-shaped hyperecho (in terms of distribution and direction) and intracranial hematoma is characterized by a mass-like hyperecho. CEUS can detect necrotic tissue in both the gunshot injured area and hematoma with more accuracy. It can also differentiate degenerative from normal or viable tissue. Ultrasound would be potentially valuable in the management of acute craniocerebral gunshot injuries and for imagingguided debridement.

\section{Conflicts of interest}

The authors declare that they have no conflict of interest.

\section{REFERENCES}

Benvenuti L, Chibbaro S, Carnesecchi S, Pulerà F, et al. (2005). Automated three-dimensional volume rendering of helical computed tomographic angiography for aneurysms: an advanced application of neuronavigation technology. Neurosurgery 57: 69-77.

Carey ME, Sarna GS, Farrell JB and Happel LT (1989). Experimental missile wound to the brain. J. Neurosurg. 71: 754-764. Cosgrove D (2006). Ultrasound contrast agents: an overview. Eur. J. Radiol. 60: 324-330.

Dawkins AA, Evans AL, Wattam J, Romanowski CA, et al. (2007). Complications of cerebral angiography: a prospective analysis of 2,924 consecutive procedures. Neuroradiology 49: 753-759.

Erdoğan N, Tucer B, Mavili E, Menkü A, et al. (2005). Ultrasound guidance in intracranial tumor resection: correlation with postoperative magnetic resonance findings. Acta Radiol. 46: 743-749.

Gervaise A, Foscolo S, Rivierre A, Derelle A, et al. (2010). Imaging of cranial gunshot traumas. J. Radiol. 91: 1113-1120.

Golea A, Badea R, Socaciu M, Diaconu B, et al. (2010). Quantitative analysis of tissue perfusion using contrast-enhanced transabdominal ultrasound (CEUS) in the evaluation of the severity of acute pancreatitis. Med. Ultrason. 12: 198-204.

He W, Jiang XQ, Wang S, Zhang MZ, et al. (2008). Intraoperative contrast-enhanced ultrasound for brain tumors. Clin. Imaging. 32: 419-424. 
Jannin P and Morandi X (2007). Surgical models for computer-assisted neurosurgery. Neuroimage 37: 783-791.

Kanno H, Ozawa Y, Sakata K, Sato H, et al. (2005). Intraoperative power Doppler ultrasonography with a contrastenhancing agent for intracranial tumors. $J$. Neurosurg. 102: 295-301.

Kim PE, Go JL and Zee CS (2002). Radiographic assessment of cranial gunshot wounds. Neuroimaging Clin. N. Am. 12: 229-248.

Kühne CA, Zettl RP, Baume B, Vogt FM, et al. (2007). Penetrating gunshot injuries to the head and brain. Diagnosis, management and prognosis. Unfallchirurg 110: 341-349.

Lee H, Wintermark M, Gean AD, Ghajar J, et al. (2008). Focal lesions in acute mild traumatic brain injury and neurocognitive outcome: CT versus 3T MRI. J. Neurotrauma 25: 1049-1056.

Lv F, Tang J, Luo Y, Li Z, et al. (2011). Contrast-enhanced ultrasound imaging of active bleeding associated with hepatic and splenic trauma. Radiol. Med. 116: 1076-1082.

Metting Z, Rödiger LA, Stewart RE, Oudkerk M, et al. (2009). Perfusion computed tomography in the acute phase of mild head injury: regional dysfunction and prognostic value. Ann. Neurol. 66: 809-816.

Mitsuya K, Nakasu Y, Horiguchi S, Harada H, et al. (2010). Perfusion weighted magnetic resonance imaging to distinguish the recurrence of metastatic brain tumors from radiation necrosis after stereotactic radiosurgery. J. Neurooncol. 99: 81-88.

Moschouris H, Malagari K, Papadaki MG, Kornezos I, et al. (2010). Contrast-enhanced ultrasonography of hepatocellular carcinoma after chemoembolisation using drug-eluting beads: a pilot study focused on sustained tumor necrosis. Cardiovasc. Intervent. Radiol. 33: 1022-1027.

Oertel M, Kelly DF, Lee JH, McArthur DL, et al. (2002). Efficacy of hyperventilation, blood pressure elevation, and metabolic suppression therapy in controlling intracranial pressure after head injury. J. Neurosurg. 97: 1045-1053.

Ract C, Le Moigno S, Bruder N and Vigué B (2007). Transcranial Doppler ultrasound goal-directed therapy for the early management of severe traumatic brain injury. Intensive Care Med. 33: 645-651.

Yetiser S and Kahramanyol M (1998). High-velocity gunshot wounds to the head and neck: a review of wound ballistics. Mil. Med. 163: 346-351.

Zimmer A and Reith W (2008). Diagnostic imaging of traumatic brain injury. Radiologe 48: 503-516. 